\title{
SMR
}

\section{Deep RNA sequencing elucidates microRNA- regulated molecular pathways in ischemic cardiomyopathy and nonischemic cardiomyopathy}

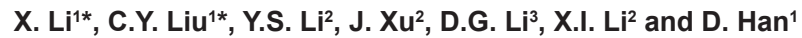 \\ ${ }^{1}$ National Center for Nanoscience and Technology, Beijing, China \\ ${ }^{2}$ College of Bioinformatics Science and Technology, Harbin Medical University, \\ Harbin, China \\ ${ }^{3}$ Institute of Biomedical Engineering, Capital Medical University, Beijing, China \\ *These authors contributed equally to this study. \\ Corresponding authors: D. Han / X. Li \\ E-mail: dhan@nanoctr.cn / lixia@hrbmu.edu.cn
}

Genet. Mol. Res. 15 (2): gmr.15027465

Received August 18, 2015

Accepted November 17, 2015

Published April 4, 2016

DOI http://dx.doi.org/10.4238/gmr.15027465

\begin{abstract}
Deregulation of cardiac miRNA gene-regulatory networks is a feature of different heart diseases, including ischemic (ICM) and nonischemic (NICM) cardiomyopathy. Here, based on the paired miRNA and mRNA expression profiles in ICM and NICM, we identified the differentially expressed miRNAs and mRNAs and the expression signatures distinguishing ICM/NICM from control samples. Furthermore, we constructed a functional miRNA network for each disease. Analysis of the topological features of these networks revealed that the Wnt signaling pathway and cell cycle (de)regulation play critical roles in the development of ICM and NICM. In addition, comparison of the miRNA and mRNA functional profiles revealed that their expression patterns in ICM and NICM differ. These findings revealed hundreds of novel heart-failurerelated miRNAs with important regulatory functions. In summary, RNA-
\end{abstract}


seq-based transcriptome profiling in the failing human heart revealed a complex transcriptional regulation associated with the disease. The newly uncovered importance of miRNAs in disease pathogenesis highlights their value as potential diagnostic and therapeutic targets.

Key words: MicroRNA; Functional network; Heart disease; Pathway; Biomarker

\section{INTRODUCTION}

Heart failure is a complex disease with a broad spectrum of molecular features. Emerging evidence across species raises the possibility that noncoding RNA (ncRNA) contributes to the development of cardiovascular disease (Papait et al., 2013; Kumarswamy et al., 2014; Xiao et al., 2014). In addition, deregulation of cardiac gene-regulatory networks (GRNs) may lead to heart disease (Louridas et al., 2010). The notion that GRNs consist primarily of protein-coding genes is somewhat premature, as the control of miRNA on GRN activity in heart failure remains elusive (Fujita and Iba, 2008).

The best-characterized ncRNAs in the heart are the miRNAs (van Rooij et al., 2008; Asrih and Steffens, 2013; Vogel et al., 2013; Flemming, 2014), that function in RNA silencing and posttranscriptional regulation of gene expression. Evidence on the role of miRNAs in cardiovascular disease is rapidly emerging. A cardiac-specific miRNA, miR-208, plays a prominent role in cardiac hypertrophy (Callis et al., 2009). In addition, miR-133a has also been implicated in cardiac hypertrophy, and mice deficient for miR-133a manifest cardiomyopathy (Carè et al., 2007). Other groups have demonstrated that miR-18b, miR-21, and miR-23a are involved in cardiomyocyte plasticity (Tatsuguchi et al., 2007). In addition, the expression of some miRNAs is altered in animal models of heart failure, as well as in human cardiac patients. Recently, a genome-wide study on miRNA expression has demonstrated that 43 miRNAs were differentially expressed in at least one of three human heart pathologies (Ikeda et al., 2007). Functional studies performed in vitro and in vivo have demonstrated key roles for miRNAs in heart failure. For instance, overexpression of miR23a, miR-195, or miR-214 induces cardiac hypertrophy in neonatal cardiomyocytes. Transgenic miR-195 mice develop dilated cardiomyopathy, while overexpression of miR-133 inhibits cardiac hypertrophy (Carè et al., 2007; Latronico and Condorelli, 2009). Altogether, these studies suggest that miRNAs play key roles in heart-related diseases and represent good therapeutic targets or have diagnostic significance in heart failure.

Cardiomyopathy can be ischemic (ICM) or nonischemic (NICM), and the expression profiles of genes or miRNAs are useful in discriminating ICMs from NICMs (Matkovich et al., 2009). A study of myocardial tissue in 17 patients showed a decrease in miR-1, -133a, and -133b in dilated cardiomyopathy patients (Ikeda et al., 2007). However, these miRNAs showed increased expression in ischemic cardiomyopathy patients. Together, these results suggest that subtypespecific miRNA expression profiles exist for cardiomyopathies of diverse etiologies. However, miRNAs are important posttranscriptional regulators that are predicted to regulate hundreds of target genes (Kolokythas et al., 2011; Jacobsen et al., 2013). The role of miRNA GRNs in different types of heart disease remains unclear. Several studies predicted the function of miRNAs based on enrichment analysis of their target genes from a number of functional categories, such as Gene Ontology (GO) and Kyoto Encyclopedia of Genes and Genomes (KEGG) pathways (Tseng et al., 2011; Li et al., 2012). These analyses provided miRNA-function profiles, aiding in the systematic 
study of miRNA function in complex diseases. However, the ICM- and NICM-specific miRNA function profiles have not been revealed.

In the study presented here, a molecular and bioinformatic pipeline was developed and optimized for the comprehensive analysis of myocardial miRNA and mRNA expression with nextgeneration sequencing. First, differentially expressed miRNAs and genes in ICM and NICM, respectively, were identified. Based on the miRNA gene regulation and functional annotation datasets, we constructed miRNA-function networks for each disease. By comparing the subtypespecific networks between ICM and NICM, common and specific miRNAs, genes, and functions were discovered. This study constructed a functional spectrum of miRNAs and dissected the irregulation in the human heart diseases ICM and NICM.

\section{MATERIAL AND METHODS}

\section{Datasets}

\section{Paired miRNA and mRNA expression profiles in ICM and NICM}

The paired miRNA-mRNA expression profiles of ICM and NICM were downloaded from gene expression and hybridization array data repositories (GEO ID: GSE46224) (Yang et al., 2014). Briefly, paired left ventricular (LV) samples from patients with severe heart failure of ICM $(\mathrm{N}=8)$ and $\operatorname{NICM}(\mathrm{N}=8)$ origin were collected at the time of LV assist device (LVAD) implantation (pre-LVAD). In addition, Human LV samples $(\mathrm{N}=8)$ were also collected from donor hearts.

The mRNA expression profiles were measured by RNA-Seq and the miRNA expression profiles were measured by small RNA-Seq. RNA sequence reads were aligned to the human genome (hg19) with TopHat (Trapnell et al., 2009), and the alignment files were reconstructed with Cufflinks (Pollier et al., 2013). For protein-coding gene analysis, the RefSeq and Ensembl transcript databases were chosen as annotation references. The read counts of each gene were normalized to the length of the gene and to the total mapped read count in each sample and expressed as reads per kb of exon per million mapped reads (RPKM). With the use of miRanalyzer (Hackenberg et al., 2011) and Bowtie (Langmead, 2010), small RNA-Seq sequence reads were mapped to the miRBase (version 18) human database for the identification of known miRNAs (Kozomara and Griffiths-Jones, 2011). The miRNA expression is presented as sequences per million mapped reads (PMMR).

\section{Ago CLIP-supported miRNA-target interactions}

To explore the miRNA-regulatory landscape, we retrieved the conserved miRNA target sites predicted by at least one of five algorithms [TargetScan (Lewis et al., 2005), miRanda (Betel et al., 2010), Pictar (Krek et al., 2005), PITA (Kertesz et al., 2007), and RNA22 (Loher and Rigoutsos, 2012)]. The combination of the predicted results with multiple-transcriptome-wide AGO-CLIP data sets has been demonstrated to result in a higher yield of high-confidence miRNA binding sites. To reduce false-positive predictions on miRNA-regulatory interactions, we next intersected the miRNA target sites, predicted with the aforementioned algorithms, with Ago-CLIP clusters to gain CLIPsupported sites. All the CLIP-identified binding site clusters or peaks were retrieved from starBase v2.0 ( $\mathrm{Li}$ et al., 2014). Using this approach, we characterized 420,000 interactions between 386 conserved miRNAs and 13,802 protein-coding genes. 


\section{Identification of differentially expressed miRNAs and mRNAs}

Differential expression analysis was performed by comparing both the miRNA and gene expression profiles in ICM or NICM with normal controls. Differential expression of genes was analyzed using paired Student $t$-tests. In addition, the differential expression of miRNAs was analyzed using the EdgeR software package (Robinson et al., 2010). The miRNAs and genes with $P$ values lower than 0.05 were considered as differentially expressed.

\section{Functional enrichment analysis of the miRNA-regulated differentially expressed mRNAs}

In order to unravel miRNA-regulatory interactions, a functional enrichment analysis was performed. Here, we selected miRNAs and genes that exhibited opposite patterns of up- or downregulation. KEGG pathways or GO terms were considered in our current study. If the whole genome had a total of $N$ genes, of which $K$ were involved in the pathway or GO terms under investigation, and the set of interesting miRNA target genes for analysis had a total of $M$ genes, of which $x$ were involved in the same pathway or GO terms, then the $P$ value can be calculated to evaluate the enrichment significance for that pathway or those GO terms according to the following equation:

$$
\mathrm{P}=1-\mathrm{F}(\mathrm{x} \mid \mathrm{N}, \mathrm{K}, \mathrm{M})=1-\sum_{\mathrm{t}=0}^{\mathrm{x}} \frac{\left(\begin{array}{l}
\mathrm{k} \\
\mathrm{t}
\end{array}\right)\left(\begin{array}{l}
\mathrm{N}-\mathrm{K} \\
\mathrm{M}-\mathrm{t}
\end{array}\right)}{\left(\begin{array}{l}
\mathrm{N} \\
\mathrm{M}
\end{array}\right)}
$$

Pathways or GO terms with P values lower than 0.05 were regarded as significant.

\section{Construction of the miRNA functional network in ICM and NICM}

After identifying all the pathways or GO terms regulated by miRNAs as described above, we constructed an miRNA-function network for ICM and NICM. In the network, the nodes represent miRNAs and their functions (pathway or GO terms), and miRNAs were linked to functions enriched by their target genes.

\section{RESULTS AND DISCUSSION}

\section{Differential miRNA and mRNA expression in ICM}

Based on the paired miRNA and mRNA expression profiles, we first identified the differentially expressed miRNAs and mRNAs in ICM. At a significance level of 0.05, 165 miRNAs and 368 mRNAs were identified to be differentially expressed (Table S1). By systematic analysis of the expression patterns of miRNAs, we found that 83 miRNAs were downregulated and 82 were upregulated (Figure 1A).

Among these miRNAs, several have been demonstrated to play key roles in heart disease. The human miRNA-21 gene is located on the plus strand of chromosome 17q23.2 and it plays an important role in the development of heart disease (Thum et al., 2008; Roy et al., 2009). It is one of the miRNAs whose expression is increased in failing murine and human hearts. Furthermore, inhibition of miRNA-21 in mice using chemically modified and cholesterol-conjugated miRNA inhibitors (antagomirs) inhibits interstitial fibrosis and improves cardiac function in a pressure- 
overload cardiac disease mouse model (Thum et al., 2008).

However, the majority of the differentially expressed genes in ICM were upregulated (306), and only 62 genes were downregulated. Hierarchical clustering analysis revealed that both differentially expressed miRNAs and genes were globally grouped into two classes. In each class, the expression levels were similar across all patient samples and controls. Unsupervised hierarchical clustering of the expression profiles of cardiac miRNAs and mRNAs revealed a distinct expression signature for the two RNA species in cardiomyopathic samples compared with controls (Figure $1 \mathrm{~B}$ and $\mathrm{C}$ ). These results suggest that failing and nonfailing $\mathrm{LV}$ can be distinguished based on their mRNA and miRNA expression profiles.

When we investigated the function of the differentially expressed genes, we found that they are mainly involved in the regulation of the voltage-gated potassium channel complex, small molecule metabolic processes, and biological processes associated with molecular binding (Figure 1C). Potassium channels sense metabolic changes and translate them to both morphological changes and secondary signaling, thus coupling the energetic status of the cell to membrane excitability. Cardiac potassium channel dysfunction is a feature of several primary cardiomyopathies, including hypertrophic cardiomyopathy and dilated cardiomyopathy (Vatta, 2010). In addition, the energy for the cardiac muscle is supplied by adenosine triphosphate (ATP), which is primarily produced through the breakdown of fatty acids (Guertl et al., 2000). Metabolic disturbances may therefore cause cardiac muscle dysfunction and result in metabolic cardiomyopathies. All these results indicate that the miRNA and gene transcriptome are significantly changed in ICM and may disturb downstream pathways.

A

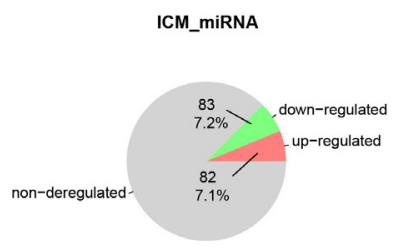

B

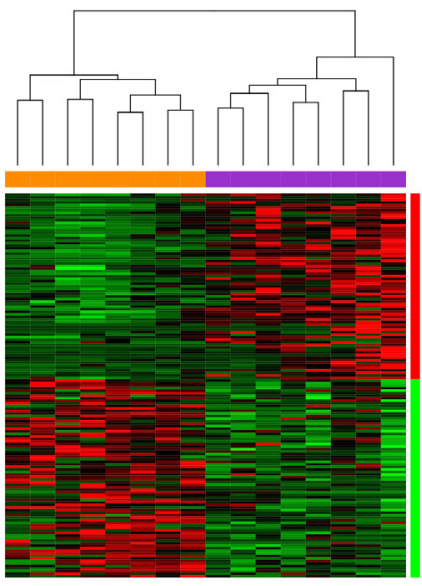

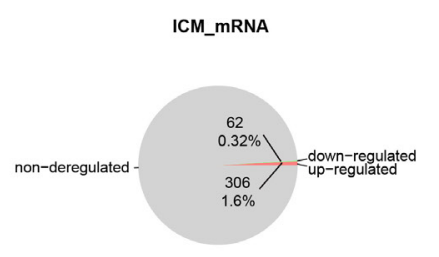

C

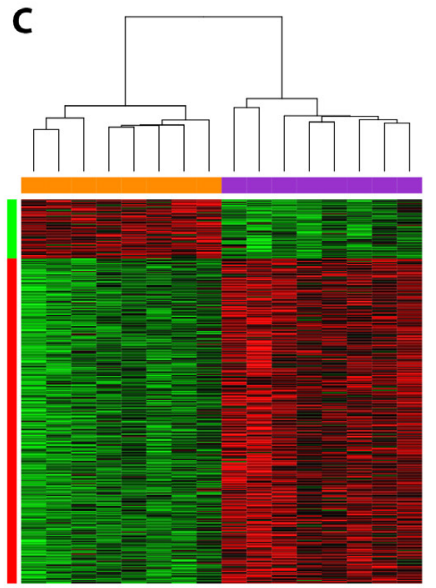

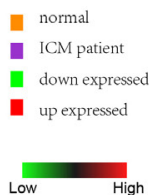

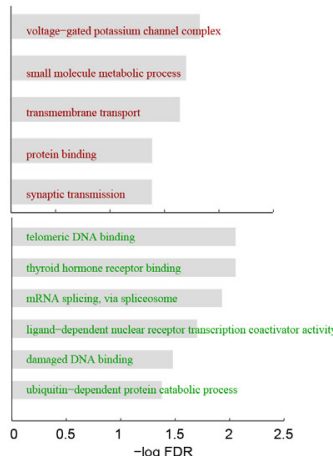

Figure 1. Differentially expressed miRNAs and genes in ischemic cardiomyopathy (ICM). A. Number of differentially expressed miRNAs and genes in ICM. B. and C. Clustering results based on miRNA and gene functions. The enriched gene functions are shown on the right in panel $\mathrm{C}$. 


\section{Functional miRNA network in ICM}

Currently, the functional interpretation of miRNAs relies mainly on the function of their target genes. Several studies predicted the functions of miRNAs based on the enrichment analysis of their target genes from a number of functional categories. The relationships between miRNAs and their regulatory targets remain poorly understood in ICM. In this study, we constructed a bipartite graph of miRNA-function interactions to explore the effects of miRNA regulation in ICM. In total, 432 miRNA-GO terms were identified, consisting of 116 miRNAs and 88 GO terms (Figure 2). The upregulated miRNAs were mainly involved in the regulation of small molecule metabolic processes, while the downregulated miRNAs are likely to play key roles in cell cycle regulation.

We also identified 74 miRNA-pathway associations in ICM (Figure 3A). The most strongly associated pathway is the Wnt signaling pathway. Many biological processes in cardiac stem cells, including cardiac specification, survival, and differentiation, are associated with Wnt signaling (Pagliari et al., 2014). We found that most of the genes in the Wnt pathway were upregulated by the downregulation of only a few miRNAs (Figure 3B), including miR-125a, miR-20a, and miR$302 \mathrm{~d}$. miR-20a inhibits the angiogenic activity of mature endothelial cells in vitro, and miR-20a knockdown increased vessel formation in an in vivo matrigel plug assay (Jakob and Landmesser, 2012). Our comprehensive analysis established an overview of miRNAs and their putative target pathways and we found that genes differentially expressed in ICM were significantly enriched for targets of certain miRNAs.

A

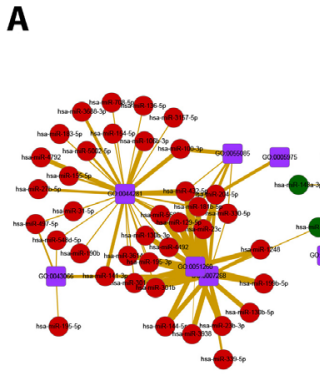

B

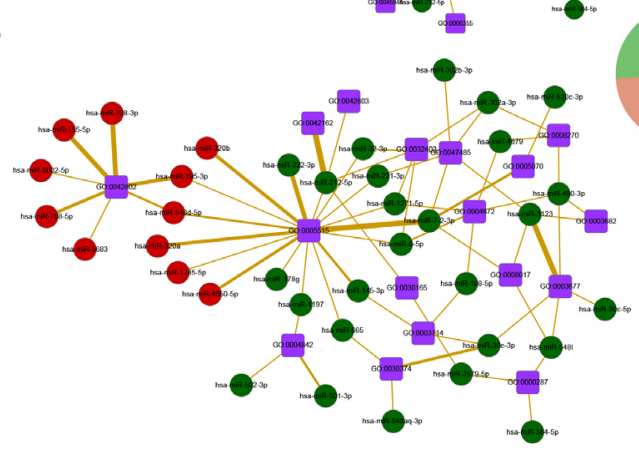

C
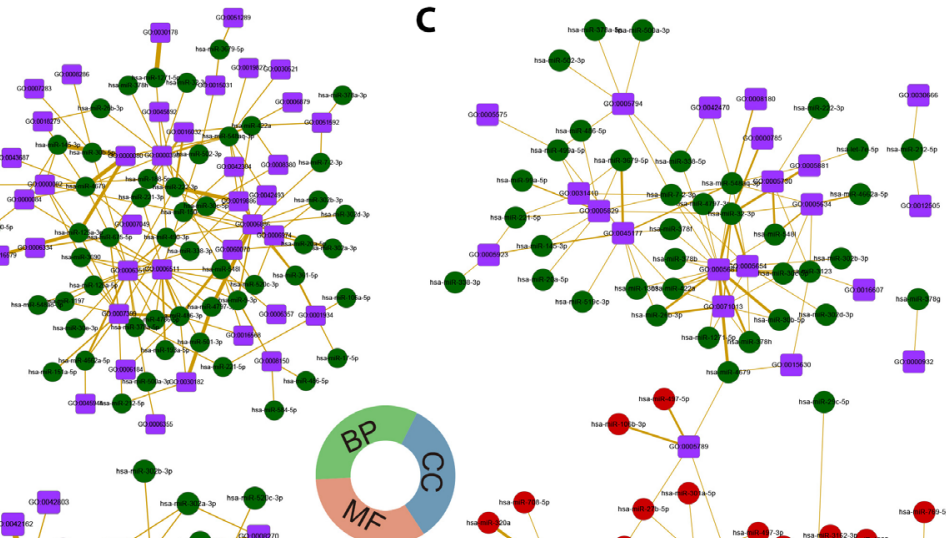

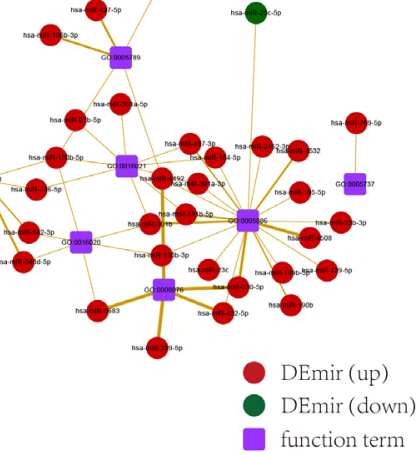

Figure 2. ICM miRNA-function network. A. Overall miRNA-biological process (BP) term networks in NICM. B. and C. miRNA-cellular components (CC) and miRNA-molecular function (MF) regulated by upregulated miRNAs and downregulated miRNAs. 

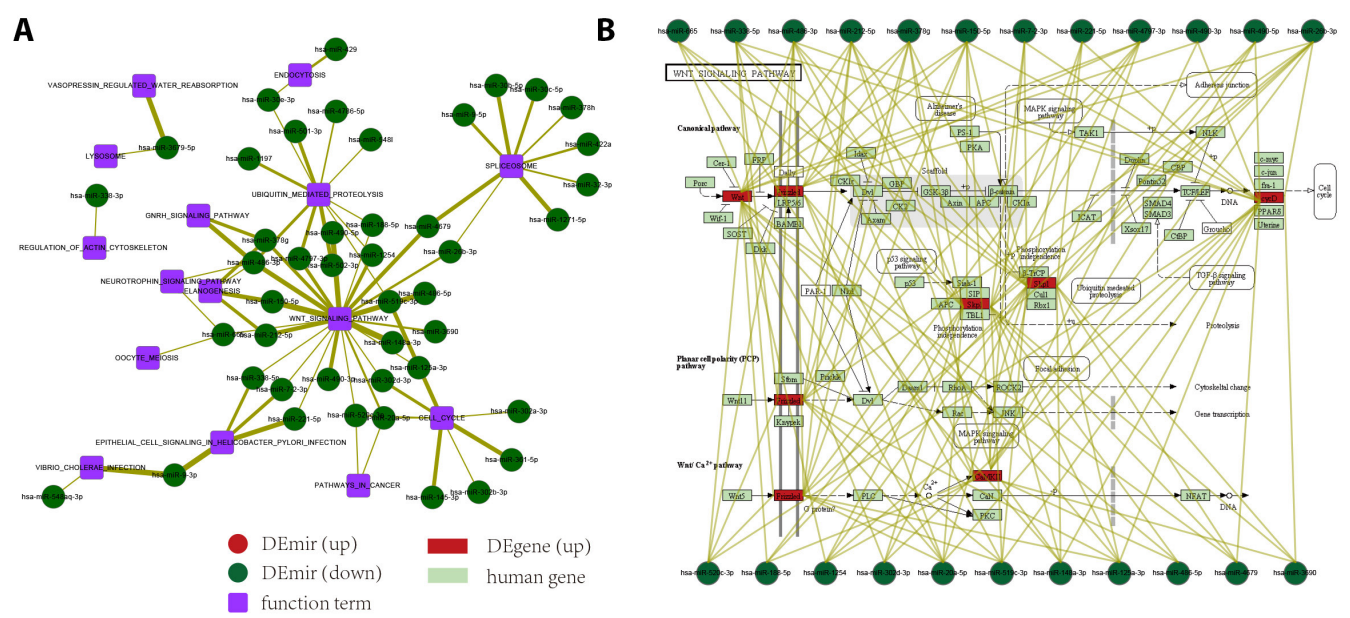

Figure 3. ICM miRNA-pathway network. A. Overall miRNA-pathway networks in ICM. B. Example of pathway regulated by downregulated miRNAs.

\section{Differential miRNA and mRNA expression in NICM}

We identified 130 miRNAs and 606 genes that showed distinct expression patterns in NICM and in control samples (Table S1). In total, 51 miRNAs were downregulated and 79 miRNAs were upregulated. In contrast, 170 genes were downregulated and 436 were upregulated (Figure $4 A$ ). One of the miRNAs that was found to be downregulated in NICM in this study was miR-17. The miR-17-92 cluster, which is induced by the transcription factor c-Myc, regulates distinct angiogenic functions (Ikeda et al., 2007). Our results demonstrate that the expression profiles of subsets of miRNAs or mRNAs are useful to discriminate ICM from control samples. To explore whether the same is true for NICM, we conducted additional analyses on subsets of miRNAs and mRNAs in NCIM. Interestingly, selection of the most differentially expressed miRNAs and mRNAs (Figure 4B and $4 \mathrm{C}$ ) resulted in discriminatory power, and NICM and control samples were more clearly separated. Functional analysis indicated that these genes are mainly involved in the regulation of small molecule metabolic processes and transforming growth factor beta (TGF- $\beta$ ) receptor binding.

\section{Functional miRNA network in NICM}

We constructed an miRNA-function networking NICM based on enrichment analysis. As a result, we found 411 significant miRNA-GO term links between 218 nodes (93 miRNAs and 125 GO terms). The most connected GO terms are 'small molecule metabolic process' and 'synaptic transmission' (Figure 5). In addition, we identified 193 miRNA-pathway pairs in NICM (Figure $6 A)$. Several of these pathways have been associated with heart disease, such as the calcium signaling and cell cycle signaling. It has been well recognized that abnormal $\mathrm{Ca}^{2+}$ handling is a key pathophysiological mechanism in human heart failure (Lou et al., 2012). However, our understanding of the molecular and cellular mechanisms underlying altered calcium handling in the failing human heart remains incomplete. Here, we provide an miRNA-gene regulatory approach to map the key roles of the calcium signaling pathway in NICM (Figure 6B). In addition, cardiac myocytes rapidly proliferate during fetal growth but exit the cell cycle soon after birth in mammals (Ahuja et al., 
2007). Cell cycle regulation is important for heart development and function. Differential miRNA expression may disturb the cell cycle via deregulation of target genes and could provide novel miRNA-based targets for NICM treatment (Figure 6C). We found that both calcium signaling and the cell cycle were regulated by a number of miRNAs that are associated with many diseases, such as $\mathrm{miR}-125$, miR-17, and miR-92.

A

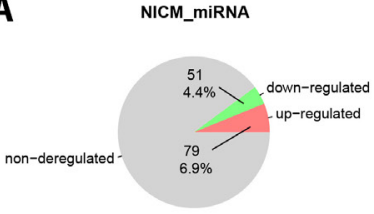

B
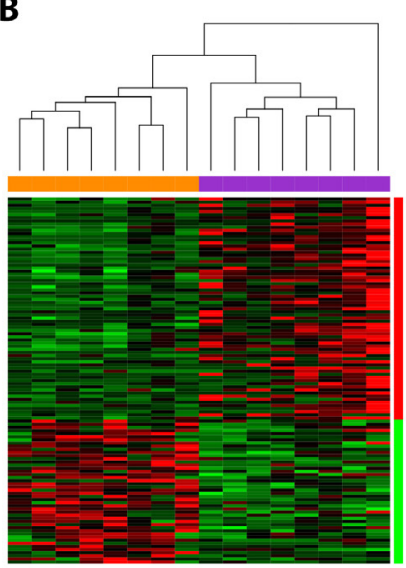

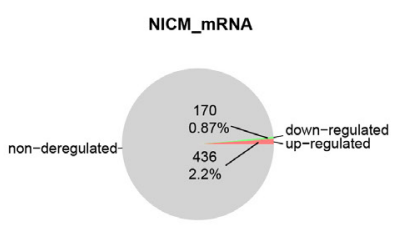

C

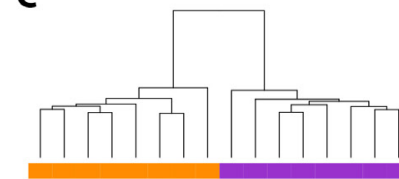

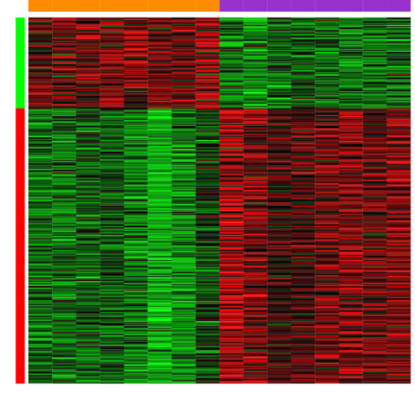

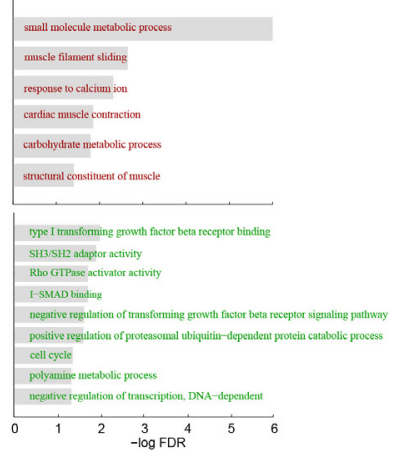

Figure 4. Differentially expressed miRNAs and genes in NICM. A. Number of differentially expressed miRNAs and genes in nonischemic cardiomyopathy (NICM). B. and C. Clustering results based on miRNA and gene functions. The enriched gene functions are shown on the right in panel $\mathrm{C}$.

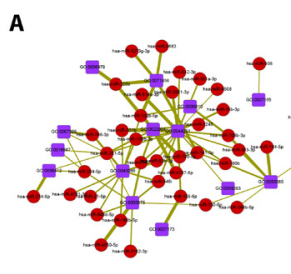

B
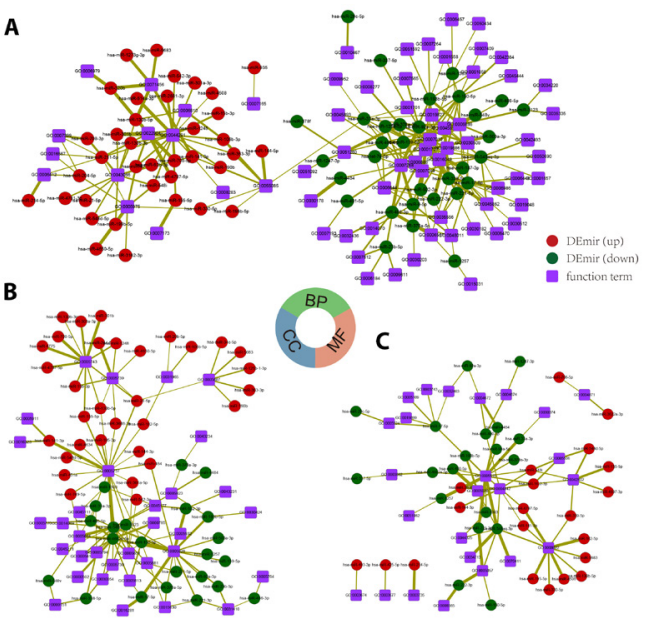

Figure 5. NICM miRNA function network. A. Overall miRNA-BP term networks in NICM. B. and C. miRNA-CC and miRNA-MF regulated by upregulated and downregulated miRNAs. 


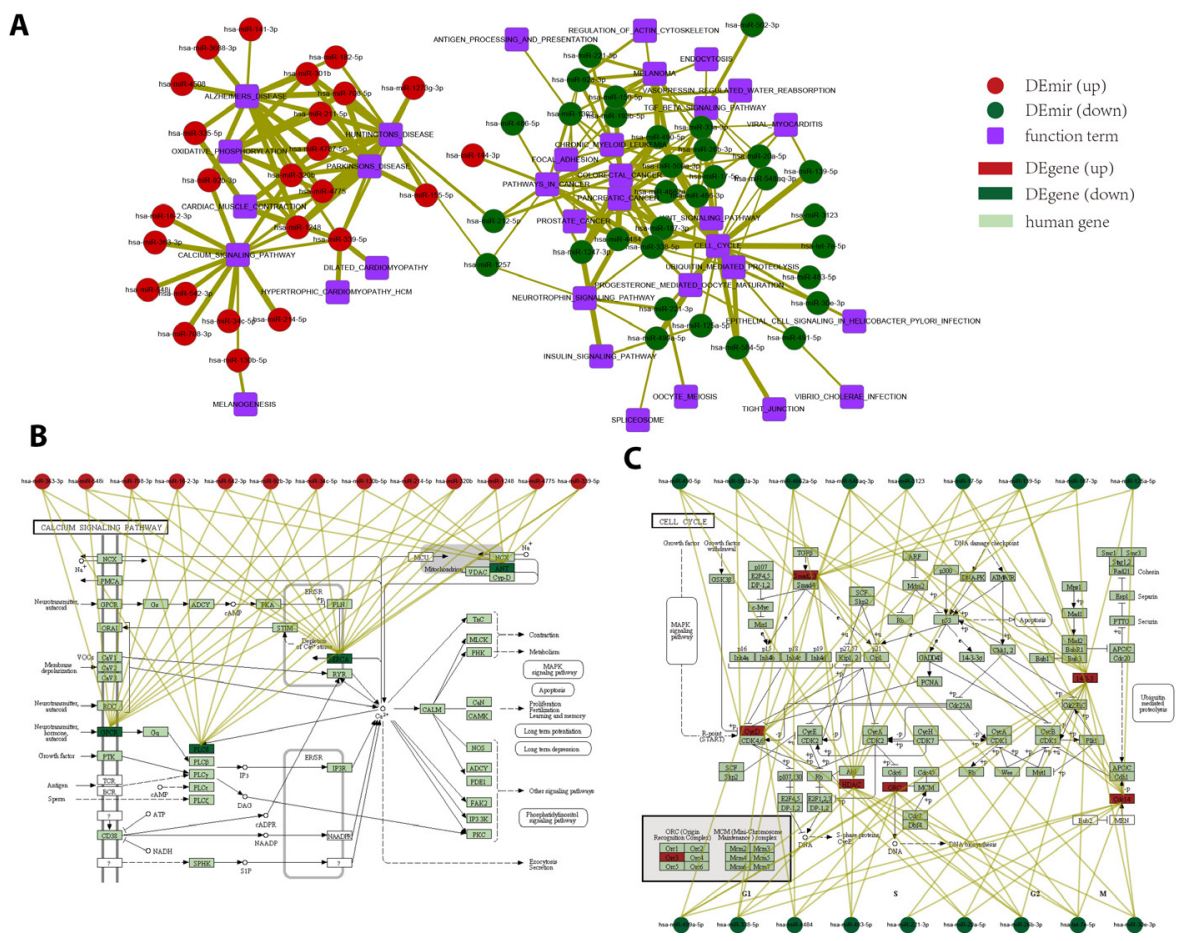

Figure 6. NICM miRNA-pathway network. A. Overall miRNA-pathway networks in NICM. B. and C. Two examples of pathways regulated by differentially expressed miRNAs.

\section{Common and specific miRNA/mRNA/functions in ICM and NICM}

The straightforward differentiation between ICM and NICM can improve disease management. Therefore, we compared the miRNAs, mRNAs, and their functions in ICM and NICM. As shown in Figure 7A and $B$, the overlap of miRNAs and genes between the two conditions was relative small. Only 55 miRNAs show consistent upregulation in ICM and NICM, including miR21-5p, miR-125b-1-3p, and miR-106b-5p. In contrast, 38 miRNAs were downregulated in both ICM and NICM, including miR-20a-5p, miR-17-5p, and let-7e-5p. In total, 180 genes showed the same differential expression pattern in ICM and NICM. Some of these genes play key roles in the heart, such as SLC9A1, ABCG2, and CCND1. CCND1 induces the expression of various genes and regulates the cell cycle and DNA replication to promote cardiomyocyte division in a rat model. Our findings also support a role for CCND1 in the heart and provide a novel insight into cell cycle control mechanisms in heart failure.

Although the ICM and NICM show a relative small overlap in their differential miRNAs and gene expression profiles, we found 41 miRNA-GO terms and 13 miRNA-pathways in both diseases. Some of these pathways play key roles in heart failure, such as the Wnt signaling pathway and the cell cycle (Figure 7C). The heart reactivates several signaling pathways and one of these pathways is the Wnt signaling pathway, which controls heart development but is also modulated during adult heart remodeling. Our current and previous studies show that the Wnt pathway plays a pivotal role in heart failure and may be a suitable target for therapeutic intervention. 
A

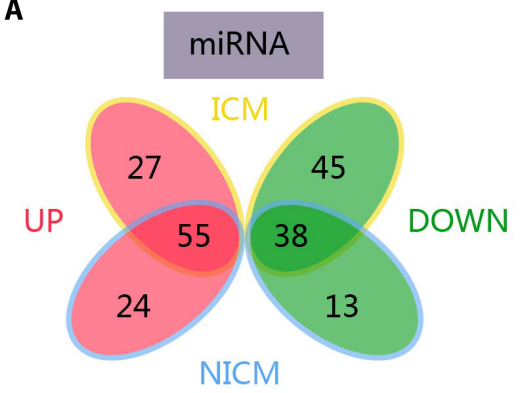

B

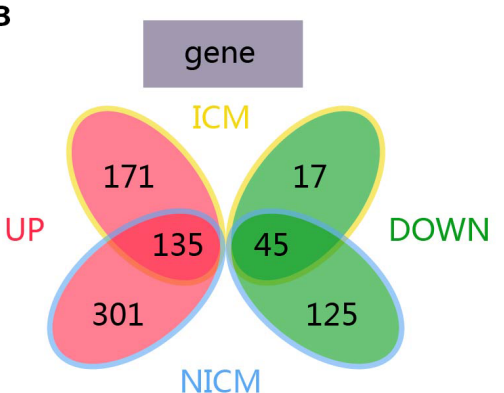

$$
\text { cell cycle }
$$

Wnt signaling pathway

ubiquitin mediated proteolysis

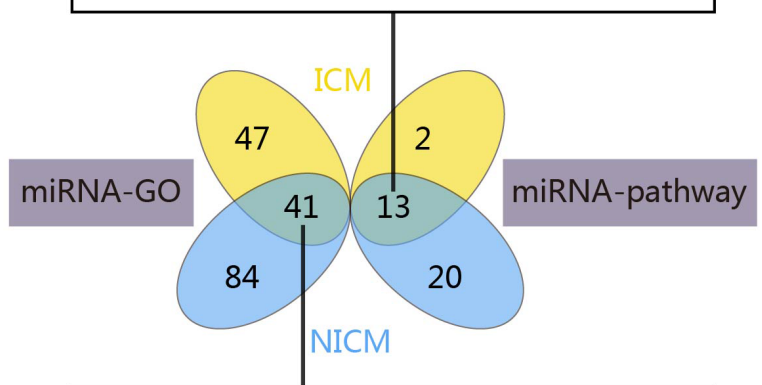

cell cycle

protein transport

synaptic transmission

stem cell maintenance

nervous system development

intracellular protein transport

ubiquitin-dependent protein catabolic process

Figure 7. Common and specific miRNA regulation in ischemic (ICM) and nonischemic cardiomyopathy (NICM). A. Venny plot showing the overlap of differentially expressed miRNAs between ICM and NICM. B. Venny plot showing the overlap of differentially expressed genes between ICM and NICM. C. Venny plot showing the overlap of miRNA and gene functions between ICM and NICM.

\section{CONCLUSION}

In summary, the myocardial miRNA and mRNA transcriptome is dynamically regulated in heart failure. The expression profiles of miRNAs or mRNAs allow for the discrimination between these different pathologies. Our results suggest that miRNAs play an important role in the pathogenesis of heart failure and the miRNAs and mRNAs identified here are attractive clinical biomarker candidates.

\section{Conflicts of interest}

The authors declare no conflict of interest.

\section{ACKNOWLEDGMENTS}

Research supported by the National Natural Science Foundation of China (\#31470905 and \#91439117) and the China Postdoctoral Science Foundation (\#2015M570985).

\section{REFERENCES}

Ahuja P, Sdek P and MacLellan WR (2007). Cardiac myocyte cell cycle control in development, disease, and regeneration. Physiol. Rev. 87: 521-544. http://dx.doi.org/10.1152/physrev.00032.2006 
Asrih M and Steffens S (2013). Emerging role of epigenetics and miRNA in diabetic cardiomyopathy. Cardiovasc. Pathol. 22: 117-125. http://dx.doi.org/10.1016/j.carpath.2012.07.004

Betel D, Koppal A, Agius P, Sander C, et al. (2010). Comprehensive modeling of microRNA targets predicts functional nonconserved and non-canonical sites. Genome Biol. 11: R90. http://dx.doi.org/10.1186/gb-2010-11-8-r90

Callis TE, Pandya K, Seok HY, Tang RH, et al. (2009). MicroRNA-208a is a regulator of cardiac hypertrophy and conduction in mice. J. Clin. Invest. 119: 2772-2786. http://dx.doi.org/10.1172/JCl36154

Carè A, Catalucci D, Felicetti F, Bonci D, et al. (2007). MicroRNA-133 controls cardiac hypertrophy. Nat. Med. 13: 613-618. http://dx.doi.org/10.1038/nm1582

Flemming A (2014). Heart Failure: Targeting miRNA pathology in heart disease. Nat. Rev. Drug Discov. 13: 336. http://dx.doi. org/10.1038/nrd4311

Fujita $\mathrm{S}$ and Iba H (2008). Gene regulatory network involving miRNA. Tanpakushitsu Kakusan Koso 53: 1781-1786.

Guertl B, Noehammer C and Hoefler G (2000). Metabolic cardiomyopathies. Int. J. Exp. Pathol. 81: 349-372. http://dx.doi. org/10.1046/j.1365-2613.2000.00186.x

Hackenberg M, Rodríguez-Ezpeleta N and Aransay AM (2011). miRanalyzer: an update on the detection and analysis of microRNAs in high-throughput sequencing experiments. Nucleic Acids Res. 39: W132-8. http://dx.doi.org/10.1093/nar/ gkr247

Ikeda S, Kong SW, Lu J, Bisping E, et al. (2007). Altered microRNA expression in human heart disease. Physiol. Genomics 31: 367-373. http://dx.doi.org/10.1152/physiolgenomics.00144.2007

Jakob P and Landmesser U (2012). Role of microRNAs in stem/progenitor cells and cardiovascular repair. Cardiovasc. Res. 93: 614-622. http://dx.doi.org/10.1093/cvr/cvr311

Jacobsen A, Silber J, Harinath G, Huse JT, et al. (2013). Analysis of microRNA-target interactions across diverse cancer types. Nat. Struct. Mol. Biol. 20: 1325-1332. http://dx.doi.org/10.1038/nsmb.2678

Kertesz M, lovino N, Unnerstall U, Gaul U, et al. (2007). The role of site accessibility in microRNA target recognition. Nat. Genet. 39: 1278-1284. http://dx.doi.org/10.1038/ng2135

Kolokythas A, Miloro M and Zhou X (2011). Review of MicroRNA Proposed Target Genes in Oral Cancer. Part II. J. Oral Maxillofac. Res. 2: e2.

Kozomara A and Griffiths-Jones S (2011). miRBase: integrating microRNA annotation and deep-sequencing data. Nucleic Acids Res. 39: D152-D157. http://dx.doi.org/10.1093/nar/gkq1027

Krek A, Grün D, Poy MN, Wolf R, et al. (2005). Combinatorial microRNA target predictions. Nat. Genet. 37: 495-500. http:// dx.doi.org/10.1038/ng1536

Kumarswamy R, Bauters C, Volkmann I, Maury F, et al. (2014). Circulating long noncoding RNA, LIPCAR, predicts survival in patients with heart failure. Circ. Res. 114: 1569-1575. http://dx.doi.org/10.1161/CIRCRESAHA.114.303915

Langmead B (2010). Aligning short sequencing reads with Bowtie. Curr. Protoc. Bioinformatics Chapter 11: Unit 11.17.

Latronico MV and Condorelli G (2009). MicroRNAs and cardiac pathology. Nat. Rev. Cardiol. 6: 419-429. http://dx.doi. org/10.1038/nrcardio.2009.56

Lewis BP, Burge CB and Bartel DP (2005). Conserved seed pairing, often flanked by adenosines, indicates that thousands of human genes are microRNA targets. Cell 120: 15-20. http://dx.doi.org/10.1016/j.cell.2004.12.035

Li JH, Liu S, Zhou H, Qu LH, et al. (2014). starBase v2.0: decoding miRNA-ceRNA, miRNA-ncRNA and protein-RNA interaction networks from large-scale CLIP-Seq data. Nucleic Acids Res. 42: D92-D97. http://dx.doi.org/10.1093/nar/gkt1248

Li X, Jiang W, Li W, Lian B, et al. (2012). Dissection of human MiRNA regulatory influence to subpathway. Brief. Bioinform. 13: 175-186. http://dx.doi.org/10.1093/bib/bbr043

Loher P and Rigoutsos I (2012). Interactive exploration of RNA22 microRNA target predictions. Bioinformatics 28: 3322-3323. http://dx.doi.org/10.1093/bioinformatics/bts615

Lou Q, Janardhan A and Efimov IR (2012). Remodeling of calcium handling in human heart failure. Adv. Exp. Med. Biol. 740: 1145-1174. http://dx.doi.org/10.1007/978-94-007-2888-2 52

Louridas GE, Kanonidis IE and Lourida KG (2010). Systems biology in heart diseases. Hippokratia 14: 10-16.

Matkovich SJ, Van Booven DJ, Youker KA, Torre-Amione G, et al. (2009). Reciprocal regulation of myocardial microRNAs and messenger RNA in human cardiomyopathy and reversal of the microRNA signature by biomechanical support. Circulation 119: 1263-1271. http://dx.doi.org/10.1161/CIRCULATIONAHA.108.813576

Pagliari S, Jelinek J, Grassi G and Forte G (2014). Targeting pleiotropic signaling pathways to control adult cardiac stem cell fate and function. Front. Physiol. 5: 219. http://dx.doi.org/10.3389/fphys.2014.00219

Papait R, Kunderfranco P, Stirparo GG, Latronico MV, et al. (2013). Long noncoding RNA: a new player of heart failure? J. Cardiovasc. Transl. Res. 6: 876-883. http://dx.doi.org/10.1007/s12265-013-9488-6

Pollier J, Rombauts S and Goossens A (2013). Analysis of RNA-Seq data with TopHat and Cufflinks for genome-wide expression analysis of jasmonate-treated plants and plant cultures. Methods Mol. Biol. 1011: 305-315. http://dx.doi. org/10.1007/978-1-62703-414-2_24 
Robinson MD, McCarthy DJ and Smyth GK (2010). edgeR: a Bioconductor package for differential expression analysis of digital gene expression data. Bioinformatics 26: 139-140. http://dx.doi.org/10.1093/bioinformatics/btp616

Roy S, Khanna S, Hussain SR, Biswas S, et al. (2009). MicroRNA expression in response to murine myocardial infarction: miR-21 regulates fibroblast metalloprotease-2 via phosphatase and tensin homologue. Cardiovasc. Res. 82: 21-29. http:// dx.doi.org/10.1093/cvr/cvp015

Tatsuguchi M, Seok HY, Callis TE, Thomson JM, et al. (2007). Expression of microRNAs is dynamically regulated during cardiomyocyte hypertrophy. J. Mol. Cell. Cardiol. 42: 1137-1141. http://dx.doi.org/10.1016/j.yjmcc.2007.04.004

Thum T, Gross C, Fiedler J, Fischer T, et al. (2008). MicroRNA-21 contributes to myocardial disease by stimulating MAP kinase signalling in fibroblasts. Nature 456: 980-984. http://dx.doi.org/10.1038/nature07511

Trapnell C, Pachter L and Salzberg SL (2009). TopHat: discovering splice junctions with RNA-Seq. Bioinformatics 25: 11051111. http://dx.doi.org/10.1093/bioinformatics/btp120

Tseng CW, Lin CC, Chen CN, Huang HC, et al. (2011). Integrative network analysis reveals active microRNAs and their functions in gastric cancer. BMC Syst. Biol. 5: 99. http://dx.doi.org/10.1186/1752-0509-5-99

van Rooij E, Marshall WS and Olson EN (2008). Toward microRNA-based therapeutics for heart disease: the sense in antisense. Circ. Res. 103: 919-928. http://dx.doi.org/10.1161/CIRCRESAHA.108.183426

Vatta M (2010). Molecular basis of arrhythmias in patients with genetically-based cardiomyopathies: when the cytoskeleton meets the ion channels. G. Ital. Cardiol. 11: 746-752.

Vogel B, Keller A, Frese KS, Leidinger P, et al. (2013). Multivariate miRNA signatures as biomarkers for non-ischaemic systolic heart failure. Eur. Heart J. 34: 2812-2822. http://dx.doi.org/10.1093/eurheartj/eht256

Xiao XG, Touma M and Wang Y (2014). Decoding the noncoding transcripts in human heart failure. Circulation 129: $958-960$. http://dx.doi.org/10.1161/CIRCULATIONAHA.114.007548

Yang KC, Yamada KA, Patel AY, Topkara VK, et al. (2014). Deep RNA sequencing reveals dynamic regulation of myocardial noncoding RNAs in failing human heart and remodeling with mechanical circulatory support. Circulation 129: $1009-1021$. http://dx.doi.org/10.1161/CIRCULATIONAHA.113.003863

\section{Supplementary material}

Table S1. Differentially expressed miRNAs and genes in ICM and NICM. http://www.geneticsmr.com/year2016/vol15-1/pdf/gmr7465 ts1.xls 